\title{
An Enhanced Virtual Time Simulator for Studying QoS Provisioning of Multimedia Services in UTRAN
}

\author{
David Soldani, Achim Wacker, and Kari Sipilä \\ Nokia Networks, System Technologies, \\ P.O. Box 301, FIN-00045 Nokia Group, Finland \\ \{david.soldani, achim.wacker,kari.sipila\}@nokia.com
}

\begin{abstract}
This paper describes an enhanced virtual time simulator for studying the provisioning of new services throughout the UMTS Radio Access Network (UTRAN). Several simulations were run to verify the feasibility of the tool and to investigate the effectiveness of the supported QoS mechanisms. The radio resources utilization is analyzed in terms of cell throughput and transmission power. The quality of experience is assessed separately for each of the offered services by tailored combinations of performance metrics, which determine the degree of satisfaction of the user of the services. The simulation results show the proposed simulator to be an appropriate tool for studying several aspects of radio network management, such as service planning and QoS provisioning.
\end{abstract}

\section{Introduction}

In UMTS only a layered bearer service architecture and QoS attributes are defined: Implementation and planning aspects of the actual QoS management functions are left to vendors' and operators' choice [1]. Hence, due to the complexity of the system and infrastructure costs, any practical deployment of radio resources management (RRM) algorithms and offered services in UTRAN needs to be validated a priori by means of static or dynamic simulations, depending on the desired level of time resolution and accuracy. For this purpose, several tools were presented in the literature, e.g.: A static simulator for WCDMA radio network planning was presented in [2], and an advanced WCDMA dynamic simulator for detailed analyses of RRM functions was described in [3]. Evaluation of the suitability and accuracy of such tools, as well as a comparison of thereof can be found in [4] and [5]. However, none of the published solutions was designed for an effective QoS provisioning. Radio network planning tools are mainly based on circuit switched (CS) communications, analyze snap shots of the system status, and do not include the possibility of handling radio resources based on QoS and/or quality of experience (QoE) requirements. Conversely, dynamic system level simulators typically run with far too high time resolution and require lengthy simulation times to design networks and/or analyze thoroughly the deployment of application services. This paper describes a virtual time simulator that overcomes the limitations of the static tool presented in [2], and the complexity of the dynamic simulator discussed in [3]. The tool enhances the version described in [6] with multi-cell propagation scenarios and realistic RRM functions as implemented in [2] and [3], respectively. Also, for more accurate analyses, the simulator includes: User definable traffic models for packet switched streaming, Push to Talk over Cellular (PoC), See What I See (SWIS, or real time video sharing, RTVS), Multimedia Messaging (MMS), WAP 
and Dialup connections [7]; and the possibility of monitoring system and service performances as recommended in [8].

Section 2 describes the simulator architecture, the currently supported traffic and propagation models, QoS management functions and metrics for assessing service and network performances. Section 3 presents the simulated environment and parameters. Section 4 examines the outputs of the simulator and discusses the performance results. Section 5 summarizes the key issues and conclusions upon the simulator utilization.

\section{Simulator Structure}

The simulator consists of a modular structure with clear interfaces. Each module is implemented independently so that each entity may be straightforwardly replaced by an alternative solution. The tool includes the following functions: Traffic and path loss generators, Admission Control (AC), Load Control (LC), Packet Scheduler (PS), Power Control (PC), Process Calls (PrC) and Performance Monitoring (PM). AC, LC and PS are cell-based algorithms, whereas PC, PrC and PM are system-based functions. The statistically large enough amount of User Equipments (UEs) in the system does not make it necessary to have them really moving: The mobility effects may be taken into account by e.g. speed dependent $\mathrm{E}_{\mathrm{b}} / \mathrm{N}_{0}$ requirements. Soft handover (SHO) affects mainly $\mathrm{AC}$ and PS. In the former, diversity (DHO) branches are processed first, followed by the main branches. In the latter, the bit rate assigned to the radio link set (UE) is the minimum of the bit rates allocated separately (for each cell) to all radio links of the active set. SHO gains may be taken into account in the $\mathrm{E}_{\mathrm{b}} / \mathrm{N}_{0}$ requirements based on SHO condition. Since the system in high traffic situations is downlink capacity limited [5], the presented simulator supports only this direction. The structure to simulate the uplink could be easily implemented using exactly the same concept except that the transmission power levels at base station (BS) would need to be replaced by the received ones. The maximum resolution of the tool is one radio resource indication period $(R R I)$, i.e. the time needed to receive the power levels from the base stations. A simulation flowchart is illustrated in Fig. 1.

\subsection{Traffic Generator, Models and Mix}

Call and session arrivals are generated following a Poisson process [8], and mapped onto the appropriate QoS profiles [1], depending on the carried type of traffic. Circuit switched (CS) speech and video calls are held for an exponentially distributed service time, and their inter-arrival periods follow exactly the same type of distribution [8]. Packet switched services are implemented as an ON/OFF process with truncated distributions [9]. The duration of the ON period depends mainly on the allocated bit rate and object size, which is modeled differently depending on the carried application [10]. Different distributions are also used to model the related OFF time behaviors and session lengths. The utilized traffic models and the adopted traffic mix (in share of calls) in this work are listed in Table 1.

All calls/sessions (generated at the beginning of each simulation) are subsequently processed (played back) taking into account the corresponding arrival times, service activities and priorities, hence the name virtual time simulator. 


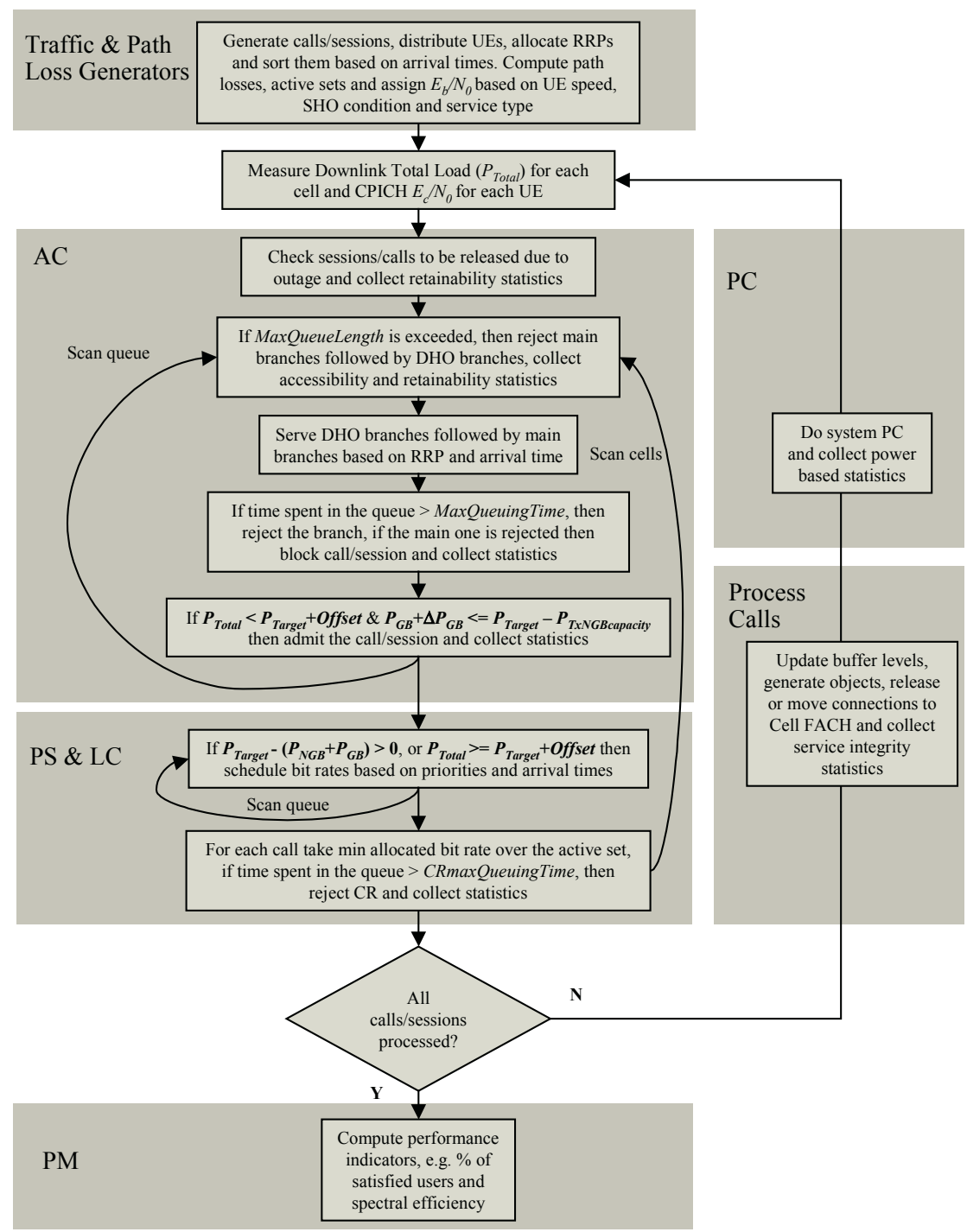

Fig. 1. Simulation flow chart.

\subsection{Path Loss Generator}

Across the simulation area, each call gets assigned a random position, but also other particular mobile distributions are possible. For each mobile location, the received power levels from all cells are calculated first and then the cells satisfying the SHO conditions are assigned as active. Each cell can be configured separately. For the path loss calculations, the Okumura-Hata model described in [11] and the models defined in [12] are supported. By implementing an appropriate interface it is also possible to import the propagation calculated by another radio network planning tool. Correlated slow fading can be overlaid as described in [8]. Fig. 2 (a) and (b) show the path losses 
Table 1. Adopted traffic models and mix.

\begin{tabular}{|c|c|c|c|c|c|c|}
\hline Service & $\begin{array}{c}\text { Data rate } \\
(\mathrm{kb} / \mathrm{s})\end{array}$ & $\begin{array}{l}\text { Buffer } \\
\text { size }(s)\end{array}$ & $\begin{array}{l}\text { Object size } \\
(\mathrm{kB})\end{array}$ & $\begin{array}{l}\text { Off time } \\
(\mathrm{s})\end{array}$ & $\begin{array}{l}\text { Session length } \\
\text { (Objects) }\end{array}$ & $\begin{array}{l}\text { Mix } \\
(\%)\end{array}$ \\
\hline $\mathrm{PoC}$ & 8 & 1 & $\begin{array}{c}\text { Exponential } \\
6 \text { mean, } \\
0.5 \text { min, } 40 \text { max }\end{array}$ & $\begin{array}{c}\text { Exponential } \\
60 \text { mean, } \\
1 \text { min, } 1200 \text { max }\end{array}$ & $\begin{array}{c}\text { Geometric } \\
8 \text { mean, } \\
1 \text { min, } 30 \text { max }\end{array}$ & 18 \\
\hline Streaming & 64 & 8 & $\begin{array}{c}\text { Uniform } \\
160 \mathrm{~min}, 3200 \max \end{array}$ & - & 1 & 12 \\
\hline MMS & $\begin{array}{l}\text { Best } \\
\text { Effort }\end{array}$ & - & $\begin{array}{c}\text { Exponential } \\
20 \text { mean, } 3 \text { min, } 200 \text { max }\end{array}$ & - & 1 & 5 \\
\hline Dialup & $\begin{array}{l}\text { Best } \\
\text { Effort }\end{array}$ & - & $\begin{array}{c}\text { Log-normal } \\
(\mu=5, \sigma=1.8) \\
0.1 \mathrm{~min}, 20000 \max \end{array}$ & $\begin{array}{c}\text { Pareto } \\
(\mathrm{k}=2, \alpha=1) \\
2 \min , 3600 \max \end{array}$ & $\begin{array}{c}\text { Inv. Gaussian } \\
(\mu=3.8, \lambda=6) \\
1 \mathrm{~min}, 50 \mathrm{max}\end{array}$ & 15 \\
\hline SWIS & 64 & 1 & $\begin{array}{c}\text { Exponential } \\
80 \text { mean, } 32 \text { min, } 2400 \text { max }\end{array}$ & - & 1 & 10 \\
\hline WAP & $\begin{array}{l}\text { Best } \\
\text { Effort }\end{array}$ & - & $\begin{array}{c}\text { Log-normal } \\
(\mu=2, \sigma=1) \\
0.1 \min , 50 \max \end{array}$ & $\begin{array}{l}\text { Exponential } \\
20 \text { mean, } \\
1 \text { min, } 600 \text { max }\end{array}$ & $\begin{array}{l}\text { Geometric } \\
3 \text { mean, } \\
1 \text { min, } 50 \text { max }\end{array}$ & 13 \\
\hline Speech & 12.3 & - & - & - & $\begin{array}{l}\text { Exponential } \\
\quad 90 \mathrm{~s}\end{array}$ & 20 \\
\hline Video & 64 & - & - & - & $\begin{array}{c}\text { Exponential } \\
120 \mathrm{~s} \\
\end{array}$ & 7 \\
\hline
\end{tabular}

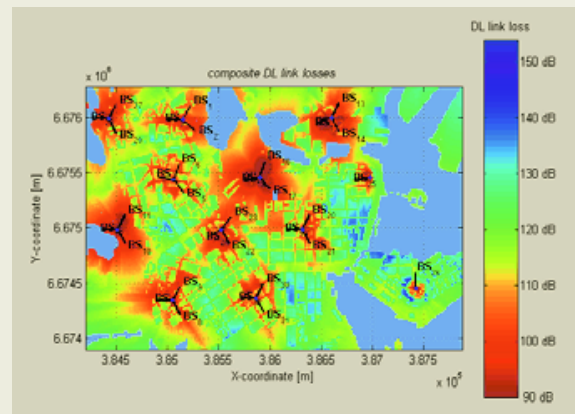

(a)

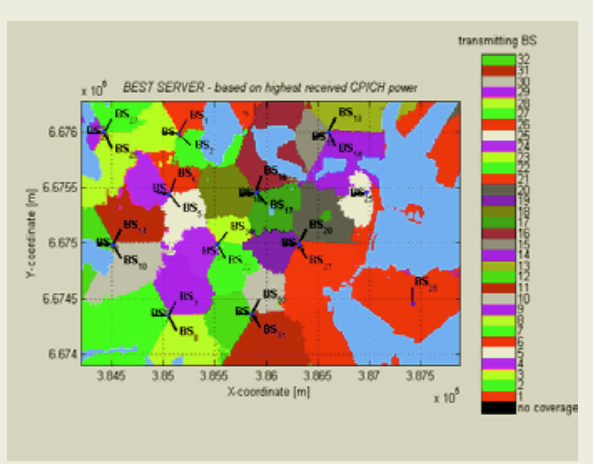

(b)

Fig. 2. (a) Example of supported link losses; (b) example of cell dominance areas.

and the cell dominance areas for the simulation scenario (downtown Helsinki, Finland) adopted in this work.

\subsection{Admission Control Function}

When a connection is set up, AC assigns to the bearer in question a Resource Request Priority $(R R P)$ value based on its QoS profile. (The lower the RRP value the higher the priority.) New radio link requests are arranged into a queue and served following the strict priority principle (branch additions have top priority) and, at a given priority, based on their arrival times (FIFO). Resource requests are rejected if either the queue length or the corresponding maximum queuing time is exceeded. Except for the overload situation, defined by

$$
P_{\text {TxTotal }}=P_{N G B}+P_{G B}>P_{\text {TxTarget }}+\text { Offset }
$$


where $P_{\text {TxTotal }}$ is the total transmission power in the cell due to guaranteed (GB) and non guaranteed bit rate (NGB) traffic and $P_{\text {TxTarget }}+$ Offset is the overload threshold, NGB bearers are always admitted; whereas GB traffic is blocked if either (1) or the following inequality is satisfied:

$$
P_{G B}+\Delta P_{G B}>P_{\text {TxTarget }}-P_{T x N G B \text { capacity }}
$$

where $P_{T x N G B c a p a c i t y}$ is the capacity optionally dedicated to NGB traffic and $\Delta P_{G B}$ is the estimated power increase in the best serving cell if the bearer in question is admitted. $\Delta P_{G B}$ is calculated using (3), which is a modified version of the formula used in [5] to estimate the initial power when a radio link is established for the first time:

$$
\Delta P_{T x}=\frac{\rho R}{W}\left(\frac{P_{t x, C P I C H}}{\rho_{c}}+(1-\alpha) P_{\text {TxTarget }}-P_{T x \text { Total }}\right),
$$

where $\rho$ and $R$ are the required $E_{b} / N_{0}$ and maximum bit rate of the bearer in question, $P_{t x, C P I C H}$ is the power of the common pilot channel, $\rho_{c}$ is the received CPICH E $\mathrm{E}_{\mathrm{c}} / \mathrm{N}_{0}$, $W$ is the chip rate $(3.84 \mathrm{Mchip} / \mathrm{s})$, and $\alpha$ is the DL orthogonality factor $(\alpha=1$ means perfect orthogonality). Ultimately, during SHO, diversity branches are not set up if the following condition is satisfied:

$$
P_{G B}+\Delta P_{G B}>P_{\text {TxTarget }}+\text { Offset },
$$

where $P_{G B}$ is the actual non-controllable power in the target cell and $\Delta P_{G B}$ is the estimated power increase in the same cell due to the radio link in question.

\subsection{Packet (Bit Rate) Scheduler Function}

Bit rates of admitted NGB bearers are scheduled based on their actual $R R P$ values and arrival times of the corresponding Capacity Requests (CRs). In the bit rate allocation, PS follows the best effort model and relies upon the power budget $P_{N G B, \text { Allowed }}$ left by the GB and NGB active $(a)$ and inactive $(i)$ connections, i.e.

$$
P_{N G B, \text { Allowed }}=P_{\text {TxTarget }}-\left(P_{G B}^{a}+P_{G B}^{i}+P_{N G B}^{a}+k \cdot P_{N G B}^{i}\right) .
$$

The power of inactive GB traffic, $P_{G B}^{i}$, takes into account the needs of the bearer services just admitted, but not yet on air; whereas the NGB inactive power, $P_{N G B}^{i}$, is the one reserved for the bearer services in discontinuous transmission (DTX). $k$ is a weighting factor, which allows the user to specify the amount of total power for inactive NGB connections to be taken into account in the power budget; $k$ ranges from 0 to 1,1 being the most conservative value. Bit rates are allocated every scheduling period by matching the estimated transmission powers to sum up to (5). Power estimates are based on (3). In the case of SHO, the allocated bit rate is the minimum of the bit rates scheduled for each of the links of the radio link set. Capacity requests are rejected if they queue longer than the corresponding $C R$ maximum queuing time. Allocated bit rates may be rescheduled only if the ongoing communication has lasted more than the related granted minimum allocation time. The bit rate allocation method for the dedicated channel is based on the minimum and maximum allowed bit 
rates, which define, respectively, the lower and upper limits of the allocated Transport Format Set (TFS). In the current implementation, only dedicated transport channels (DCHs) are available for packet data transmission. Bearers that were longer in DTX than the corresponding inactivity timer are moved to Cell FACH (Forward Access Channel). In this state, the transmission is temporarily interrupted. When new data arrives at the radio network controller (RNC) buffer, a new CR is sent to PS and subsequently another DCH is allocated to the bearer in question, as explained above.

\subsection{Load Control Function}

The only load control action supported by the simulator is the reduction of bit rates of NGB bearer services when (1) is satisfied. The bit rate may be downgraded only when the allocation time of the carried service lasted longer than the corresponding granted overload minimum allocation time. The bit rates are reduced starting from the bearers with lowest priority and, at given priority, based on their arrival times (FIFO). Power estimates in the bit rate decrease algorithm are based on (3).

\subsection{Process Calls Function}

All active calls in the system are processed at once each radio resource indication period, as illustrated in Fig. 1. If the ongoing connection is CS, the simulator collects its throughput, increases the active connections counter, and releases the call in the case it lasted longer then the corresponding call duration period (see Table 1). For each packet switched connection, we check first whether either the RNC buffer or the source buffer is not empty. Then, if there is data to transmit, the active session throughput is collected and the active connections counter accordingly increased. Besides this, the status of the corresponding buffer in the UE is monitored and updated. If during the ON period the buffer gets empty, the re-buffering procedure is activated and the user of the service is considered unsatisfied. When the user is reading (or the connection is in idle mode, in the case of $\mathrm{PoC}$ ), the transfer delay of the delivered object is calculated, the connection is marked as inactive and the inactivity period monitored. If the dwelling time of the $\mathrm{DCH}$ in question lasted longer than the corresponding inactivity timer, the terminal is moved to Cell FACH state, and the corresponding allocated resources are released. When the reading time is over, either a new object to be downloaded and the corresponding reading time are regenerated, or the ongoing packet communication is released, depending on the corresponding session length (see Table 1). In the former case, if the time needed to fill up the buffer in the terminal is more than the corresponding buffering delay, the user of the service in question is recorded as unsatisfied.

\subsection{Power Control Function}

The simulator supports an ideal power control function that includes the effects of a large-scale propagation channel (see Section 2.2), but not fast fading. Multi-path fading and SHO effects are taken into account in the service $E_{b} / N_{0}$ requirement. The interference is realistically modeled: At any simulation time step, the received power from all cells except from the best server is counted as interference, and hence the corresponding coupling effect is fully taken into account. (Note: Form the best server 
only the fraction of the power determined by the non-orthogonality is considered as interference.)

The power control system of equations can be written as:

$$
\begin{gathered}
\frac{W p_{i_{m}} / L_{m, i_{m}}}{R_{i_{m}} P_{m} / L_{m, i_{m}}\left(1-\alpha_{i_{m}}\right)+\sum_{n, n \neq m} P_{n} / L_{n, i_{m}}+N_{i_{m}}}=\rho_{i_{m}}, \\
i_{m} \in I(m), \quad m=1, \ldots, M
\end{gathered}
$$

where the symbols in (6) are explained in Table 2.

Table 2. Symbols in the PC system of equations.

\begin{tabular}{cl}
\hline Symbol & Explanation \\
\hline$I_{m}$ & Index of a UE served by BS $m$ \\
$m, n$ & Indices of BSs \\
$I(m)$ & Set of UE indices served by BS $m$ \\
$M$ & Number of cells \\
$p_{i_{m}}$ & BS transmitted power for UE $i_{m}$ \\
$P_{m}, P_{n}$ & Total transmit power of BS $m$ and BS $n$ \\
$L_{m, i_{m}}$ & Pathloss from BS $m$ to UE $i_{m}$ served by BS $m$ \\
$L_{n, i_{m}}$ & Pathloss from BS $n$ to UE $i_{m}$ served by BS $m$ \\
$R_{i_{m}}$ & Bit rate used by UE $i_{m}$ \\
$\alpha_{i_{m}}$ & Orthogonality factor for UE $i_{m}$ \\
$N_{i_{m}}$ & Noise power (thermal plus equipment) of UE $i_{m}$ \\
$\rho_{i_{m}}$ & Required $E_{b} / N_{0}$ for UE $i_{m}$ \\
\hline
\end{tabular}

Equation (6) simply equates the received $E_{b} / N_{0}$ with given transmission powers to the required $E_{b} / N_{0}$ for sufficient quality of the connection. Taking into account that:

$$
P_{m}=\sum_{i_{m} \in I(m)} p_{i_{m}}+p_{c, m},
$$

where $p_{c, m}$ is the sum of common channel powers from $B S_{m}$, (6) can be rewritten in the compact form as an $M$ times $M$ linear system of equations of the type $A x=b$, where the unknowns are the total BS powers. During the simulations, we first resolve this linear system and then from (6) we derive the individual radio link powers. The solutions are then used to estimate the transmission powers of GB and NGB services in (1)-(5). AC and PC ensure that the BS total transmission power is kept below its maximum and the WCDMA pole capacity is not exceeded. The existence of solutions to the type (6) of equations was studied e.g. in [13]. The power of common channels is a cell based management parameter (see Section 3 ).

\subsection{Performance Monitoring Function}

Several performance indicators can be collected during the measurement period, e.g. call block ratio (CBR) caused by queuing and/or buffer overflow, call drop ratio (CDR) due to power outage, active session throughput (AST), capacity request rejec- 
tion ratio (CRRR) for NGB traffic, object transfer delay for Browsing, MMS and Dialup connections, and re-buffering for streaming, PoC and SWIS applications. Link and cell based powers, as well as $\mathrm{CPICH} E_{c} / N_{O}$ values are also computed during the simulated time. From such measurements is derived the geometry factor $(G)$, defined as the ratio between the received power from the serving cell and the power received from the surrounding cells plus noise [5].

System and service performances can be assessed as recommended in [8], and for this purpose tailored user satisfaction criteria can be input to the simulator. In this work, a speech or video user is satisfied if the call neither gets blocked nor dropped. In addition to this criterion, for PoC, SWIS and streaming users no re-buffering is allowed during the communication, and the time to fill up the related buffer needs to be reasonably short; for Dialup (http, emails, ftp, etc...), WAP browsing, and MMS, the AST has to be higher than $64 \mathrm{~kb} / \mathrm{s}, 32 \mathrm{~kb} / \mathrm{s}$ and $8 \mathrm{~kb} / \mathrm{s}$, respectively. Furthermore, none of the capacity requests of NGB services must be rejected. The spectral efficiency is computed as the system load (cell throughput normalized with respect to the chip rate, $3.84 \mathrm{Mchip} / \mathrm{s}$ ) at which a certain percentage of users of the worst performing service are satisfied. Different thresholds can be set for the distinct bearers, though $90 \%$ is the default value for all applications.

\section{Simulation Environment and Assumptions}

Speech, video and SWIS were offered with guaranteed bit rate, and all other services were run on the best effort. Speech and video calls were served as CS-Conversational, whereas SWIS was carried on PS-Streaming class. PoC, streaming and WAP/MMS were mapped onto PS-Interactive. Dialup connections, which comprised, for example, ftp, emails and http traffic, were carried on PS-Background class. The RRP values were set such that speech calls had top priority, followed by video and SWIS calls. Within the Interactive class, using different traffic handling priorities (THPs), PoC was handled first, followed by streaming and WAP/MMS. Dialup was served in the end. The differentiated parameter values, which further improve $\mathrm{PoC}$ and streaming performance at the expenses of lower priority services, and the mapping of the services onto distinct QoS profiles are illustrated in Table 3. Performance results were analyzed using a macro cellular network located in the downtown of Helsinki (see Fig. 3), where terminals were uniformly randomly distributed, but not on the water. The simulation was performed over a period of 2 hours using a time step of $200 \mathrm{~ms}$ (RRI period), and all statistics were collected over the entire simulation period. The traffic mix and the traffic intensity were held constant, i.e. 2 call/session attempts per second. The corresponding offered traffic was about 750 users per cell over the all simulated time. Table 4 reports the most important network parameters.

Table 3. Mapping of services onto QoS classes and parameter values.

\begin{tabular}{|c|c|c|c|c|c|c|c|c|c|c|}
\hline QoS Profile & Service & $\begin{array}{c}\text { Bit Rate } \\
(\mathrm{kb} / \mathrm{s})\end{array}$ & RRP & $\begin{array}{l}\text { Min. All. Bit } \\
\text { Rate }(\mathrm{kb} / \mathrm{s})\end{array}$ & $\begin{array}{l}\text { AC Max. } \\
\text { Queuing } \\
\text { Time (s) }\end{array}$ & $\begin{array}{c}\text { Granted Min. } \\
\text { DCH Alloc. } \\
\text { Time (s) } \\
\end{array}$ & $\begin{array}{c}\text { Granted Min. } \\
\text { DCH Alloc.Time } \\
\text { in Overload (s) }\end{array}$ & $\begin{array}{c}\text { Buffering } \\
\text { Delay } \\
\text { (s) }\end{array}$ & $\begin{array}{c}\text { Inactivity } \\
\text { Timer } \\
\text { (s) }\end{array}$ & $\begin{array}{l}\text { CR Max. } \\
\text { Queuing } \\
\text { Time (s) }\end{array}$ \\
\hline \multirow[t]{2}{*}{ CS-conv. } & Speech & 12.2 & 1 & GB & 5 & - & - & - & - & - \\
\hline & Video & 64 & 2 & GB & 10 & - & - & - & - & - \\
\hline PS-stream. & SWIS & 64 & 3 & GB & 10 & - & - & 5 & - & - \\
\hline \multirow[t]{3}{*}{ PS-int. } & PoC & 0,8 & 4 & 8 & 15 & 15 & 10 & 4 & 60 & 4 \\
\hline & Streaming & 0,64 & 5 & 64 & 15 & 10 & 5 & 16 & 5 & 10 \\
\hline & WAP/MMS & $0,16,32,64,128,144,256,384$ & 6 & 32 & 15 & 5 & 0.2 & - & 10 & 10 \\
\hline PS-backg. & Dialup & $0,16,32,64,128,144,256,384$ & 7 & 16 & 15 & 1 & 0.2 & - & 5 & 5 \\
\hline
\end{tabular}




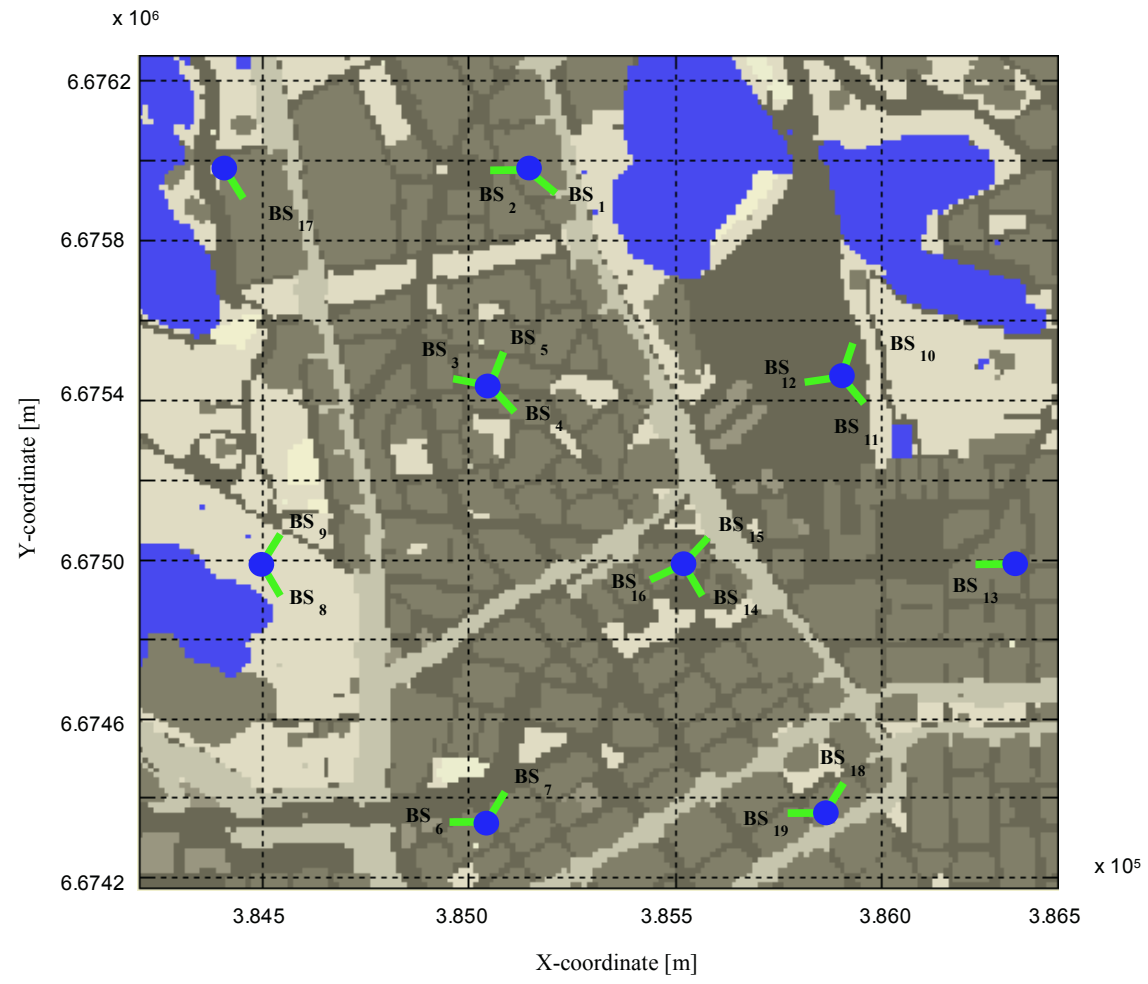

Fig. 3. Simulation scenario used in the case study discussed in this paper.

Table 4. Most important system based parameters.

\begin{tabular}{|c|c|}
\hline Parameter & Value \\
\hline Call/session mean arrival rate & $0.5 \mathrm{~s}$ \\
\hline Radio resource indication period $(R R I)$ & $0.2 \mathrm{~s}$ \\
\hline Simulation time (s) & $7200 \mathrm{~s}$ \\
\hline Power target for DL AC & $3 \mathrm{~dB}$ below BTS total power \\
\hline Overload offset for DL AC & $1 \mathrm{~dB}$ above power target \\
\hline Orthogonality $(\alpha)$ & 0.5 \\
\hline Period for load control actions & $0.2 \mathrm{~s}(1 \mathrm{RRI})$ \\
\hline Period for Packet Scheduling & $0.2 \mathrm{~s}(1 \mathrm{RRI})$ \\
\hline \multicolumn{2}{|l|}{$E_{b} / N_{0}$ requirements } \\
\hline Speech & $7 \mathrm{~dB}$ \\
\hline SWIS & $6 \mathrm{~dB}$ \\
\hline Streaming & $6 \mathrm{~dB}$ \\
\hline PoC & $7 \mathrm{~dB}$ \\
\hline MMS/WAP & $5 / 5.5 \mathrm{~dB}$ \\
\hline Dialup & $5.5 \mathrm{~dB}$ \\
\hline Maximum BTS Tx power & $43 \mathrm{dBm}$ \\
\hline P-CPICH Tx power & $33 \mathrm{dBm}$ \\
\hline Sum of all other CCH Tx powers & $30 \mathrm{dBm}$ \\
\hline Length of $\mathrm{AC}$ queue & 10 Radio bearers \\
\hline Dedicated NGB capacity & $0 \mathrm{~dB}$, i.e. not used \\
\hline Power weight for inactive NGB traffic $(k)$ & 0.5 \\
\hline
\end{tabular}




\section{Simulation Results and Discussion}

The simulation results of the case presented in the previous section are shown through Fig. 4 to Fig. 8 and in Table 5. The offered traffic mix over the all simulated period is illustrated for each of the simulated cells in Fig. 4 (a). Fig. 4 (b) illustrates the average cell throughput as a function of the deployed cells and services. From these graphs, taking into account the traffic models and mix reported in Table 1, we conclude that the traffic generator works as planned.

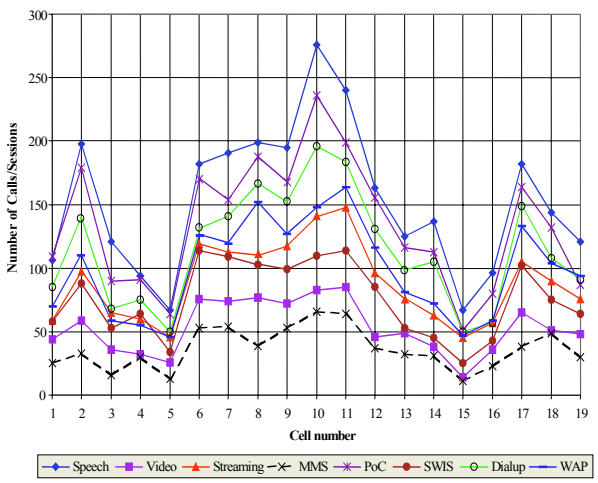

(a)

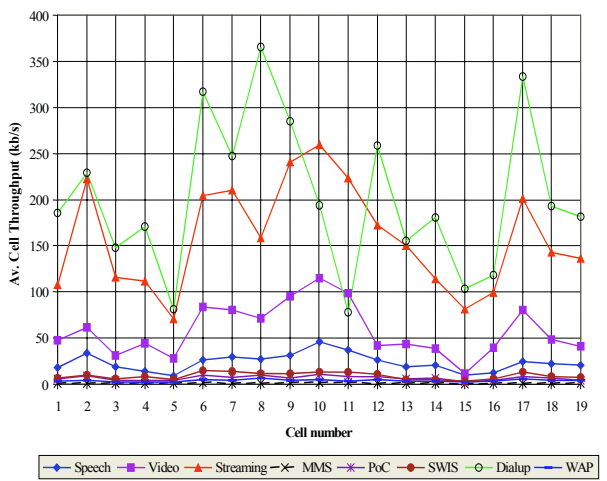

(b)

Fig. 4. Traffic distribution over the 19 cells: (a) Offered load in call arrivals; (b) average cell throughputs (service based values in $\mathrm{kb} / \mathrm{s}$ for each of the simulated cells).

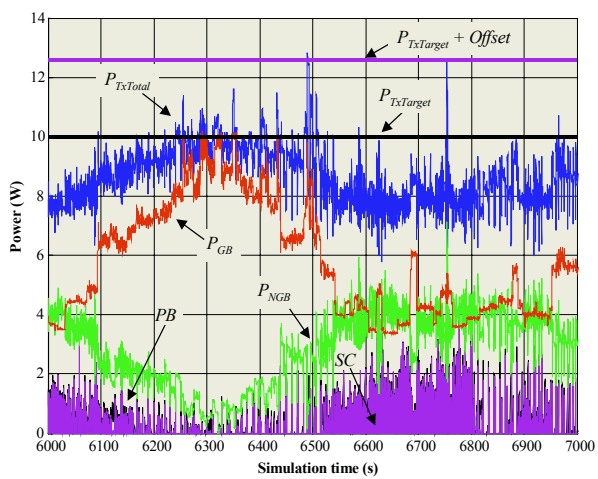

(a)

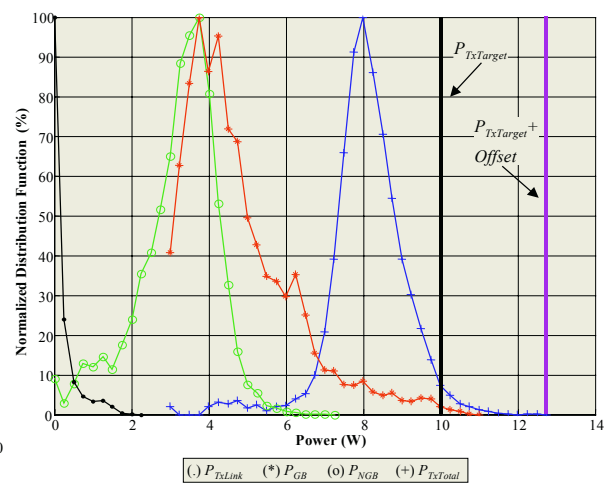

(b)

Fig. 5. Cell 11: (a) Snapshot of the simulation period; (b) normalized distribution functions of the radio link (.), GB (*), NGB (o), and total (+) downlink transmission power.

A snapshot (1000 s) of the load status in Cell 11 (see Fig. 3), where most of the users resulted unsatisfied, is depicted in Fig. 5 (a). The values plotted against the simulation time are the transmission powers, the power budget $(P B)$ and scheduled capacity $(S C)$. From this figure, we can notice that all supported QoS management functions work as intended. In fact, the power budget for NGB traffic, defined in (5) 
and denoted by $P B$ in the figure, is properly scheduled, i.e. at a given NGB traffic volume and available bit rates, the curve of the allocated bit rates $(S C)$ closely follows the $P B$ one. Hence, the scheduling algorithm presented in Section 2.4 turns out to perform as well as expected. Besides this, when the total load in the cell $\left(P_{\text {TxTotal }}\right.$, i.e. $P_{G B}+P_{N G B}$ ) reaches the overload threshold, denoted by $P_{\text {TxTotal }}+$ Offset, the NGB allocated bit rates are accordingly reduced by LC and in turn immediately resumed when the $P_{G B}$ decreases and the system backs off to its normal state of operation. Furthermore, the measured GB load $\left(P_{G B}\right)$ hardly ever trespasses the target threshold $\left(P_{\text {TxTarget }}\right)$ defined in (2), which confirms the accuracy of (3) in the power estimates during the admission control and bit rate allocations. Ultimately, following the input traffic mix, $P_{\text {TxTotal }}$ preserves its point of equilibrium $\left(P_{\text {TxTarget }}\right)$, which thus validates the supported AC and PC functions.

The normalized distribution functions of the transmission powers in Cell 11 are shown in Fig. 5 (b). The radio link power $\left(P_{T x L i n k}\right)$, GB transmission power $\left(P_{G B}\right)$, NGB transmission power $\left(P_{N G B}\right)$ and total downlink transmission power $\left(P_{T x \text { Total }}\right)$ distributions reflect exactly the load status in the cell previously discussed, the input parameter values reported in Table 4 , and the constraints in the RRM functions defined in Section 2.3, 2.4, 2.5 and 2.7.

None of the calls/sessions was rejected due to buffer overflow and almost none of the speech, video and SWIS calls was blocked due to the time spent in the AC queue, as shown in Table 5 in terms of call block ratio (CBR). This is in line with the offered traffic, which hardly ever exploited the available cell capacity. As a result, almost all users of the GB services resulted satisfied.

The effects of the prioritization between GB services can be noticed in Fig. 6 (a), where the percentage of satisfied users for each of the deployed services is illustrated as a function of the simulated cells. In Cell 10 and 11, the quality experienced by speech users, in terms of CBR, is better than the accessibility offered to SWIS and video users. As expected, more evident in the figure is the differentiated treatment of NGB traffic, which reflects exactly the provisioned discrimination between real time (RT) and non-real time (NRT) services of Interactive and Background classes. In fact, in each of the simulated cells, the percentage of satisfied users of Dialup is the lowest, followed by WAP and MMS; and for the RT services, PoC performance is always better than the streaming one.

A more detailed analysis upon the reason why the users of NGB services were not satisfied is possible based on the raw performance indicators illustrated in Fig. 6 (b), Fig. 7, and in Fig. 8 (a). In these figures, the intended differentiation between services in terms of the metrics characterizing the QoE of each of the deployed services is also visible. In particular, Fig. 6 (b) and Fig. 7 (a) show, respectively, the $10^{\text {th }}$ percentile of the average active session throughput (AST) and the capacity request rejection ratio (CRRR) collected for each of the above services during the measurement period, as a function of the deployed cells. The throughput experienced by Dialup users is lower than the corresponding one offered to WAP/MMS users, which underwent the same treatment. Conversely, the accessibility offered to PoC and streaming services, while requesting capacity to PS, is better than the corresponding blocking experienced by WAP/MMS and Dialup users. Fig. 7 (b) reveals how the throughput deterioration adversely affects the PoC and streaming performance in terms of re-buffering in the UE. As expected, the re-buffering ratio is higher for streaming, whereas the dissatis- 
faction due to "too long time to refill up the buffer" depicted in Fig. 8 (a) is higher for PoC. This is due to the fact that the tolerance for streaming users (up to sixteen seconds) was higher than for PoC ones, which were not supposed to wait for more than four seconds (see Table 3 ).

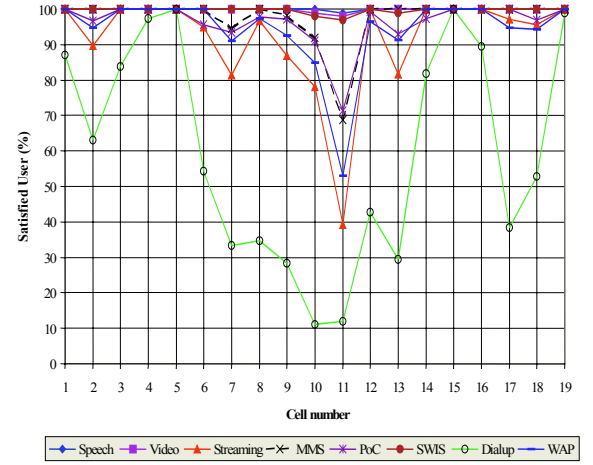

(a)

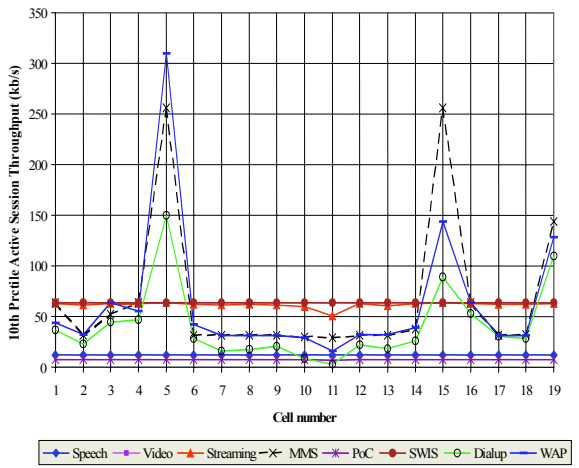

(b)

Fig. 6. Service based indicators for each of the simulated cells: (a) Percentage of satisfied users; (b) $10^{\text {th }}$ percentile of the average active session throughput during the simulated time.

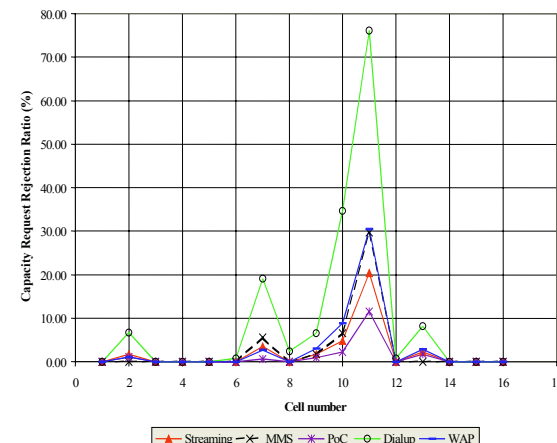

(a)

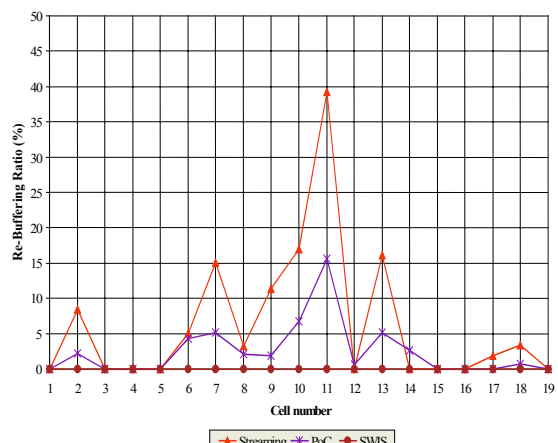

(b)

Fig. 7. Service based performance indicators for each of the simulated cells: (a) Capacity request rejection ratio; (b) re-buffering ratio.

The differentiation between WAP/MMS and Dialup connections and the benefit of thereof is also shown in Fig. 8 (b) and Table 5, where the transfer delays of the WAP, MMS and Dialup objects during the simulation period are presented. The measured metrics reflect exactly the calculated object delay from the median of the AST and object size, which is an additional evidence that the process call and performance monitoring functions (see Fig. 1, Section 2.6 and 2.8) work as intended.

\section{Conclusions}

An effective solution to study the provisioning of QoS before the deployment of new services throughout UTRAN was presented. As a part of this framework, the currently 
supported QoS management functions were investigated in terms of: Offered traffic mix, transmission powers, throughput, queuing time, object transfer delay, call block ratio, call drop ratio, capacity request rejection ratio, and percentage of satisfied users. Simulation results showed the proposed virtual time simulator to perform as expected and to be a good trade off between the complexity of an advanced dynamic simulator and the straightforwardness of a quasi-static tool. Thus, the described solution has the potential for investigating any QoS management algorithm and multimedia service provisioning in UTRAN, before its deployment throughout a real WCDMA network. Furthermore, the simulator may be used to find an optimum trade-off between service quality, capacity and coverage requirements for any of the services in a $3 \mathrm{G}$ mobile network operator's service portfolio.

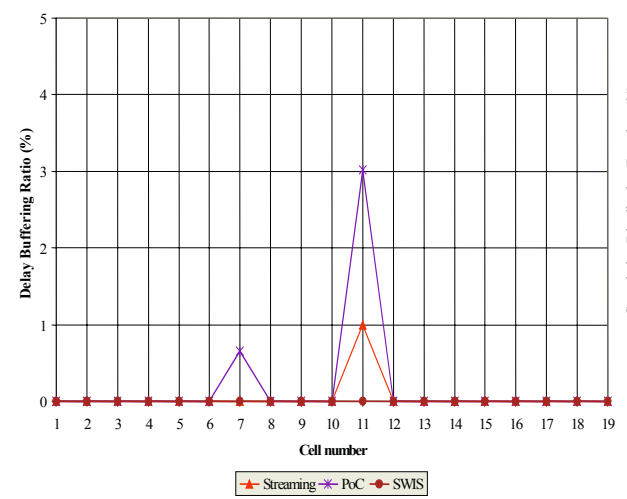

(a)

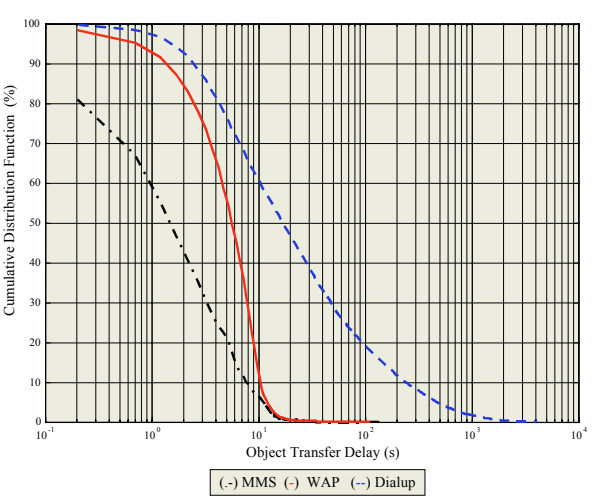

(b)

Fig. 8. (a) Too long time needed for re-buffering ratio (service based performance indicators for each of the simulated cells); (b) MMS, WAP and Dialup object transfer delays (system based statistics upon all simulated time).

Table 5. System based measurement results.

\begin{tabular}{lccccccccc}
\hline $\begin{array}{c}\text { Service } \\
\text { type }\end{array}$ & $\begin{array}{c}\text { CBR } \\
(\%)\end{array}$ & $\begin{array}{c}\text { CDR } \\
(\%)\end{array}$ & $\begin{array}{c}\text { CRRR } \\
(\%)\end{array}$ & $\begin{array}{c}\text { RBR } \\
(\%)\end{array}$ & $\begin{array}{c}\text { DBR } \\
(\%)\end{array}$ & $\begin{array}{c}\text { Median } \\
\text { AST } \\
(\mathrm{kb} / \mathrm{s})\end{array}$ & $\begin{array}{c}\text { Median } \\
\text { Object } \\
\text { Size } \\
(\mathrm{kB})\end{array}$ & $\begin{array}{c}\text { Calculated } \\
\text { Object } \\
\text { Delay }\end{array}$ & $\begin{array}{c}\text { SU } \\
(\%)\end{array}$ \\
\hline Speech & 0.05 & 0.00 & - & - & - & 12.2 & - & - & 99.95 \\
Video & 0.16 & 0.00 & - & - & - & 64.0 & - & - & 99.84 \\
Streaming & 0.00 & 0.00 & 1.93 & 6.35 & 0.05 & 63.4 & 1682 & 212.2 & 91.67 \\
MMS & 0.00 & 0.00 & 2.29 & - & - & 70.5 & 15 & 1.7 & 97.55 \\
PoC & 0.00 & 0.03 & 1.08 & 2.49 & 0.19 & 8.0 & 4 & 4.0 & 96.31 \\
SWIS & 0.32 & 0.00 & - & 0.00 & 0.00 & 64.0 & 89 & 11.1 & 99.68 \\
Dialup & 0.00 & 0.00 & 8.44 & - & - & 51.4 & 120 & 18.7 & 59.94 \\
WAP & 0.00 & 0.05 & 2.57 & - & - & 66.0 & 48 & 5.8 & 94.24 \\
\hline
\end{tabular}

Note: $\mathrm{RBR}=$ Re-Buffering Ratio, DBR = Delay Buffering Ratio; SU = Satisfied Users 


\section{References}

1. 3GPP, TS 23.107: QoS Concept and Architecture

2. Wacker, A., Laiho, J., Sipilä, K., Jäsberg, M.: Static Simulator for Studying WCDMA Radio Network Planning Issues. Proceedings of VTC, IEEE, Vol.3. Spring (1999) 2436-2440

3. Hämäläinen, S., Holma, H., Sipilä, K.: Advanced WCDMA Radio Network Simulator. Proceedings of PIMRC, IEEE. Aalborg, Denmark (1999) 509-604

4. Holma, H., Toskala, A. (eds.): WCDMA for UMTS. John Wiley \& Sons, Revised Edition (2001) $313 \mathrm{p}$

5. Laiho, J., Wacker, A., Novosad, T. (eds.): Radio Network Planning and Optimization for UMTS. John Wiley \& Sons (2002) 484 p

6. Soldani, D., Laiho, J.: A Virtual Time Simulator for Studying QoS Management Functions in UTRAN. Proceedings of VTC, IEEE, Vol. 5. Fall (2003) 3453-3457

7. Halonen, T., Romero, J., Melero, J. (eds.): GSM, GPRS and EDGE Performance. John Wiley \& Sons, Second Edition (2003) $615 \mathrm{p}$

8. ETSI, TR 101112 (UMTS 30.03) v.3.2.0: Selection Procedures for the Choice of Radio Transmission Technologies of the UMTS

9. Shankaranarayanan, N., Jiang, Z., Mishra, P.: User-Perceived Performance of WebBrowsing and Interactive Data in HFC Cable Access Networks. Proceedings of ICC, IEEE, Vol. 4. (2001) 1264-1268

10. Klemm, C.L., Lohmann, M.: Traffic Models for Characterization of UMTS Networks. Proceedings of GLOBECOM, IEEE, Vol. 3. (2001) 1741-1746

11. COST 231, TD(91)73: Urban Transmission Loss Models for Mobile Radio in the 900 and $1800 \mathrm{MHz}$ Bands

12. ITU-R M. 1225: Guidelines for Evaluation of Radio Transmission Technologies for IMT2000. (1997)

13. Hanly, S.V.: Information Capacity of Radio Networks. Ph.D. Dissertation, Kings College, University of Cambridge (1993) 225 p 